\title{
Research on the Inheritance and Development of Tianjin Traditional Folk Custom Pattern Yangliuqing New Year Paintings in Urban Landscape Design
}

\author{
Shiyun Zheng ${ }^{1}$ Xiaojuan Hui ${ }^{1, *}$ \\ ${ }^{1}$ Nankai University Binhai College, Tianjin 300270, China \\ *Corresponding author.Email: teresahui@foxmail.com
}

\begin{abstract}
The urban landscape of each city should serve as the carrier of local culture, publicize and better the essence of local culture, and promote the progress and development of local culture. Only the culture rooted in the local soil can highlight the essential attributes of the city in a region and enable the city to develop culturally in a healthy and orderly manner. If the regional culture of a city is not respected or even artificially dispersed, then the connection between modernity and history will be abandoned. Such urban development, even if it seems economically prosperous, brings forth cities without soul. Starting from the elements and images of Yangliuqing New Year paintings, this paper analyzes the extraction, reconstruction and application of pattern elements to realize the integration between traditional culture and modern materials. Such method promotes the inheritance of traditional culture, enhances people's sensory experience of the paintings, and makes landscape design a new way of cultural communication, so as to foster cultural confidence. The relationship between Yangliuqing New Year paintings and Tianjin urban landscape design has also been expounded in this paper, providing insight for urban landscape design in the new era.
\end{abstract}

\section{Keywords: folklore patterns, inheritance and development, modernization}

\section{INTRODUCTION}

Yangliuqing New Year paintings are very rich, and many elements are involved in their traditional patterns. Different ways can be used to express the same content, and diversified means of expression can provide space for urban landscape architects to play and present. It's presumed to convey both connotation and interest. However, designers value profit over understanding. They pursue speed and make simple substitutions, resulting in repetitive expressions and excessive similarity. For example, after the sculpture was adopted, other designers followed suit and all engaged in sculpture for a while. As a result, all creation will be overshadowed by the lack of creativity, and the public will be aesthetically tired, while the inheritance of traditional culture will be hindered.

Art originates from life. Derived from the life of ordinary people in Tianjin, Yangliuqing New Year paintings first serve the real existence and permeate into every aspect of life. However, modern urban landscape architects have artificially built a spiritual "fence"

*Fund: The paper is supported by 2019 Tianjin Municipal College Students' Innovation and Entrepreneurship Training Program Project. (Project No.: 201913663047) between people and art in the early stage of design, so that the two can only see each other from a distance, let alone interact. Some designs even adopted "guardrails," elevating them to "works of art," which is contrary to the original intent. Through in-depth and careful research, the designer effectively combines the original functions with the decorations. Not only the decorations are reflected, but also the participants can understand the original roles and functions from the interaction. Then the design makes the memory and impression more profound

By exploring the design method of "inheritance and innovation", this paper creates modern landscape design that is compatible with national culture and regional culture and integrated with the surrounding environment, to promote the national culture and improve the public aesthetic. The design and construction of modern urban landscape should shoulder the function and responsibility of improving urban and public cultural environment to continuously better the quality of public life. In doing so, the function of urban landscape is further expanded based on regular functions to cultivate public's aesthetic view. In urban construction projects, unique cultural connotations are created, and practical functions are organically integrated to display the urban style. New techniques 
and expressions are adopted to reinterpret the pattern of Yangliuqing New Year paintings and carry them forward. Tradition is no equal to antique. It should boast its own vitality in the new era. However, the full play of its vitality requires creators to continue to innovate in practice, which is also the direct meaning of this paper's attempt to realize the innovation practice of traditional art.

\section{OVERVIEW AND RESEARCH ON YANGLIUQING NEW YEAR PAINTINGS IN TIANJIN}

\section{A. Pattern characteristics of Yangliuqing New Year paintings}

1) Modeling: The Woodcut New Year pictures are quaint, simple, and concise, which not only capture the main objective characteristics of the images, but also make bold deductions, exaggerations, and deformations. It is rich in artistic style, simple in expression technique, bold, and vivid. This is mainly reflected in the randomness of the image, complete and perfect shape, in abstraction and inclusiveness.

2) Color: The color of Yangliuqing New Year paintings is warm, auspicious with strong contrast, which helps the viewer to comprehend the color of the original appearance, and the paintings combine exaggeration and random thinking. The use of color is very standard, reflecting a simple, sincere, warm feeling, a thorough reflection of the creator's feelings.

\section{B. Pattern classification of Yangliuqing New Year paintings}

The themes of Yangliuqing New Year paintings fall in three camps:

1) The picture or statue of a god or the Buddha: The material of the theme mainly comes from Zen, Taoism, Confucianism, god in folklore and the founder of the lineage.

2) Children and beauties: Children and beauties embody people's desire for a perfect family, full of children and grandchildren, healthy body, and a happy life. The combination of its color patterns is usually ideal and perfect.

3) Drama story: The theme of the drama story occupies a relatively high proportion in the New Year's patterns, which has been recognized by the public. Most of these New Year's pictures are based on historical stories, folklore, romantic novels, and operas from other places.

\section{APPLICATION OF TIANJIN YANGLIUQING NEW YEAR PAINTINGS IN LANDSCAPE DESIGN}

\section{A. Landscape design principles}

1) The combination of inheritance and innovation: The aesthetic and cultural value of Yangliuqing New Year paintings will gain new vitality through the form of modern urban landscape, so certain design principles are required. The combination of inheritance and timeliness is more than bringing Yangliuqing New Year paintings into modern design, but to combine two key elements, which requires higher understanding of cultural and artistic characteristics and local characteristics. The first is to extract and integrate the modeling elements of the paintings. The design methods such as simplification, decomposition, exaggeration, addition and subtraction and abstraction are employed to extract and innovate the shape according to the original shape, while the most traditional part and innovation should be preserved. The second is the inheritance and continuation of artistic concepts. The traditional Chinese aesthetics advocating "image" can only deeply explore the cultural connotation of patterns of Yangliuqing New Year painting. The inheritance and renewal of modern design concept can extend its "image" into landscape design, creating modern landscape art with "form and spirit". The third is modern composition. Thanks to modern composition methods such as composition displacement, proportion composition, balanced composition and appropriate composition, New Year painting patterns are incorporated into modern landscape design in a new composition form to achieve the unity of locality and timeliness.

2) The combination of art and technology: Artists in each period face different living conditions, express varied feelings, values, and visual forms, and use diverse art materials, techniques, and tools to reproduce new art forms. The perfect art form requires technology as the foundation and combines the two. Artistic value is at the core of everything. As to whether the production is exquisite, technology is the guarantee of perfect presentation.

3) The combination of regionality and timeliness: The design and creation of modern urban landscapes should rely on modern art and craftsmanship to reflect unique regional culture. It indicates social needs while keeping pace with the times. In pursuing internationalization, the finding of a way that ensures the continuous development of one's own unique culture and the harmonious development of surrounding environment is a common problem faced by all social strata. The designer should take an objective and 
comprehensive view of the landscape of the area he is dealing with guarantee the harmony between its natural appearance and history and culture.

\section{B. Evolution in landscape design}

The evolutionary design of Yangliuqing patterns in the complicated urban landscape mainly aims to study the essence of traditional culture, inherit the outstanding cultural connotation, and create sparks between contemporary art and traditional culture. It's a must to seize the opportunity of the design of Yangliuqing New Year paintings to express the unique image of the city and to translate the regional cultural symbols into the design language. This is a long process of development and improvement. And it will ultimately embody the resonance between tradition and modernity. Designers who choose repetitive manufacturing and copy instead of pursuing the connotation and form, will bring about repeated "construction" and waste of large amounts of resources, which seriously deviates from the law of art development.

The authors take that urban landscape designers should fully study and understand the true meaning of the Yangliuqing New Year painting patterns, and express it with contemporary artistic techniques. The level and ability of modern technology combine the two to achieve a high spiritual connotation and a perfect cultural external form.

1) Direct reference: Traditionally, both the planar and the three-dimensional model contain deep cultural connotation and have strong aesthetic significance in form. The appearance of these symbols will form a strong visual impact and constantly attract people's attention. However, because of its repeated appearance and use, an inherent image has been formed in people's cognition, which has become a mark. The direct reference here is not to copy the original style and expression, but to select some traditional styles with typical characteristics and meaning. The specific environment around the landscape, design orientation and functional orientation should be taken into account. The direct reference to the pattern of Yangliuqing New Year paintings requires a close combination of practicality and decoration. This can inspire people to integrate into specific environments and participate in the artistic conception of landscape design. In turn, it resonates with the audience and is recognized by all. In urban landscape design, direct reference is a convenient and effective method, but attention should be paid to avoid excessive and no consideration of reference. Direct references shorten the sense of distance between traditional culture and the masses, and make it easier for the public to accept. In this way, the masses can intuitively feel the charm and value of traditional art. (See "Fig. 1")

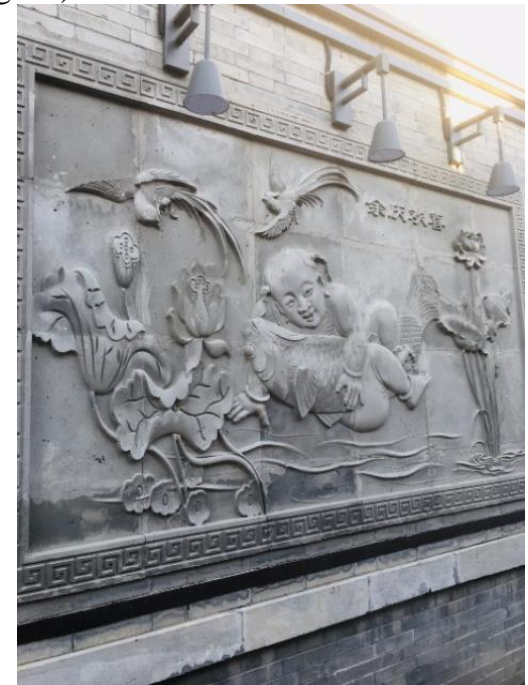

Fig. 1. Direct application of Yangliuqing New Year painting patterns.

2) Reorganization and reconstruction of graphics: Any pattern or symbol is a combination of points, lines, and faces. When deconstructing the pattern of Yangliuqing New Year painting, the original geometric figures are transformed and exaggerated through deformation and exaggeration, the delicate parts are retained, the characteristic parts are enhanced, and new elements are added to make the design look novel and new meanings are given. The application of redesigned and created graphics in landscape design can create a new cultural atmosphere with both traditional and new era characteristics. The reorganization and reconstruction of shapes is a new trend in urban landscape design in recent years, which promotes the personalized and diversified development of art forms.

3) Spatial switching: The traditional planar image is combined with three-dimensional sense through sculpture modeling. The plane and elevations are interspersed with each other organically, which not only has the aesthetic feeling of plane image, but also has three-dimensional contour. Tradition and modernity blend perfectly. The process of spatial switching reflects the flow and transformation of space. The change of spatial orientation can cause sharp contrast between reality and reality on the color, shape, pattern, and texture of the landscape's materials. The scenery and the surrounding environment form a visual space of sharp contrast, and there are rich changes, forming a mysterious beauty. 


\section{Expression in landscape design}

1) Color: The importance of color in urban landscape design is self-evident. Only by properly dealing with the color relationship between the environment and the landscape, can people better express the "emotion" conveyed by the landscape, rationally use the urban space, and make the landscape both influential and coordinated with the urban environment. The tones of traditional patterns are usually primitive. Well used and matched, they retain a strong and intuitive visual effect, thus enabling the landscape environment to display local characteristics and reflect the traditional original style. For example, in public entertainment venues, bright yellow is usually employed, which is warm and vibrant. Red is festive and enthusiastic; while purple is elegant and mysterious. In the environment as communities and parks, fresh green is adopted to indicate the peace of ecology, environmental protection, and tranquility. In short, the bold and harmonious color configuration will give the original ecological colors of traditional patterns a new look.

2) Modeling: The "modeling" is the interpretation of verbs, which refers to the process by which humans create physical images. It is also an interpretation of terms, which refers to the images of physical images created by humans in production and living practices. From the perspective of pattern design, modeling can refer to the graphics created by a combination of points, lines, and faces, while from that of landscape design, modeling, the external form of landscape design, is the most intuitive element. For traditional decorative patterns or landscape designs, whether they are flat patterns or physical objects with spatial shapes, certain modeling rules must be followed in the design. The style of most modelling is natural, simple, lively, easy, and have distinctive region characteristic, which shows people's desire for a better life.

In modern landscape design, modeling elements of folk graphics are often used, such as paper-cut, New Year painting, woodcut picture, drama, seal, calligraphy, Chinese knot, etc. These traditional graphic elements have strong national characteristics. Designers use these modeling elements to perfect, process, combine, reshape, and other artistic techniques to create works with national and contemporary characteristics that convey auspicious meanings, such as completeness, happiness, good luck, and longevity.

3) Texture: When a designer creates a work, if the physical properties and symbolic meanings of the materials used are consistent with the deep connotation of the work, it will be more suitable for the theme and content, endowing the work a vivid and stronger artistic charm. The textures of materials have their own attributes and temperament, which will bring people different aesthetic feelings and emotional needs. Although only a medium, it plays an extremely important role in landscape design and creation. Reasonable selection and combination of materials make the urban landscape present a variety of styles and often determine its quality. Designers are required to consider different themes and shapes, both culturally and historically, both functionally and aesthetically. Therefore, the design of urban landscape involves various factors, which requires designers to study history and culture. In investigating the needs of the audience, they also need to understand the characteristics of various materials extensively and pinpoint whether they can accurately convey the intention of the audience. Any kind of material is naturally or artificially endowed with a certain sense of beauty and function, which is unique and attractive by itself. Proper collocation can not only enrich language expression, but also better reflect aesthetic value and social function. As a result, the exploration of the charm and characteristics of the material itself is conducive to the discovery of materials with expressive functions, so that they can coexist harmoniously with the surrounding environment and interact with each other, thus showing the charm of the environment and landscape, which is an interactive relationship. The patterns of New Year paintings are various and can be expressed in various forms. Different styles can adapt to different scenes. Therefore, in landscape design, the proper selection and combination of materials can maximize landscape and environment. (See "Table I")

TABLE I. MATERIALS AND PROPERTIES

\begin{tabular}{|l|l|l|}
\hline Material & \multicolumn{1}{|c|}{ Implication } & \multicolumn{1}{|c|}{$\begin{array}{c}\text { External } \\
\text { features }\end{array}$} \\
\hline Wood & Oriental, soft, flexible & $\begin{array}{l}\text { Environmental } \\
\text { protection, } \\
\text { nature }\end{array}$ \\
\hline Bamboo & $\begin{array}{l}\text { Longevity, happiness, } \\
\text { spiritual truth }\end{array}$ & Nature, green \\
\hline Metal & Technology & Extensibility \\
\hline Glass & Light, meticulous & $\begin{array}{l}\text { Transparent, } \\
\text { light }\end{array}$ \\
\hline $\begin{array}{l}\text { Ceramic } \\
\text { tile }\end{array}$ & Traditional, delicate & Shiny, crisp \\
\hline Plastic & Modern, generous & Flexible \\
\hline Stone & Eternity, commitment & $\begin{array}{l}\text { Hard, varied in } \\
\text { shape }\end{array}$ \\
\hline $\begin{array}{l}\text { Brick } \\
\text { and tile }\end{array}$ & $\begin{array}{l}\text { Standing still, the sense of } \\
\text { age, since time immemorial }\end{array}$ & $\begin{array}{l}\text { Strong } \\
\text { catching effect }\end{array}$ \\
\hline$\ldots$ & $\ldots$ & $\ldots$ \\
\hline
\end{tabular}




\section{Design carriers in landscape design}

The patterns of Yangliuqing New Year paintings are colorful and varied. These artistic elements embody the wisdom of the working people and reflect the higher aesthetic level accumulated in people's life, which is enough to endow the landscape with profound connotation. The full use of traditional patterns in Tianjin's urban landscape should not only emphasize the appreciation of the regional image it represents, but also consider the interaction between people and the city, human and nature, practical and artistic functions.

1) Public guidance system: The public guidance system refers to the use of designed text or graphics in public areas to indicate directions and area identification. It is characterized by accuracy, easy identification, and memory. It exists in the urban environment and is therefore greatly influenced by the space and environment in which it is located. It is also affected by many factors, such as the appearance, location, and materials of the building. It not only has the elements of visual communication art, but also influences architectural design, environmental design, and other aspects. As independent disciplines, they interact and overlap. The correct, comprehensive, and systematic application of the New Year picture design requires the painstaking efforts of designers. (See "Fig. 2")

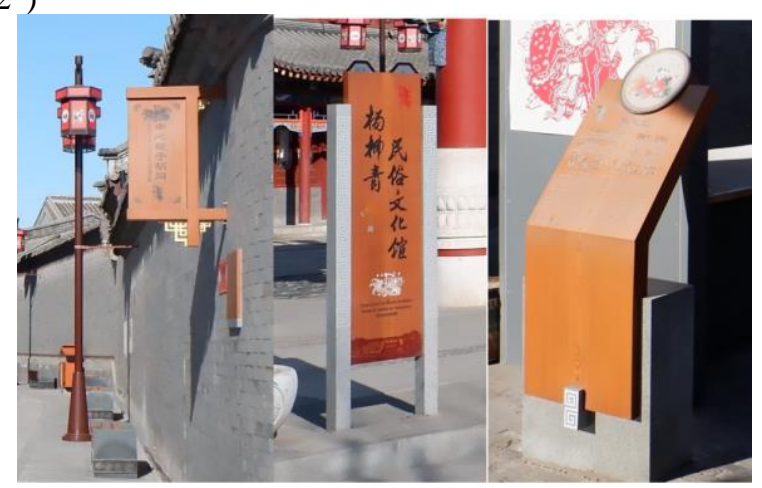

Fig. 2. Street lamp, street sign and attraction sign designed with the elements of Yangliuqing New Year paintings.

2) Pavement: The pavement in the urban landscape enjoys a large proportion, high frequency of use, and desirable effect. It is the most common form of urban landscape. Good pavement brings convenience and a clean environment for everyone. The use of patterns on the ground began during the Ming and Qing dynasties, when various patterns that reflected the ingenuity of ancient craftsmen for the pavement of the gardens are created. In the long process of practice, they found different permutations and combinations. These patterns, both interesting and artistic, have been passed down from generation to generation, evolving, passing on, innovating, and improving. Different materials and patterns can be applied to pavements and recreational trails. The patterned shapes and different colors can be employed to decorate roads according to regional characteristics and main functions. In the design, the shape of traditional auspicious patterns can be directly referred to, or local patterns can be used. The gap in the ground or conventional decoration will greatly increase the characteristics of the local landscape, while the trail landscape will greatly enhance the cultural connotation. (See "Fig. 3" and "Fig. 4")

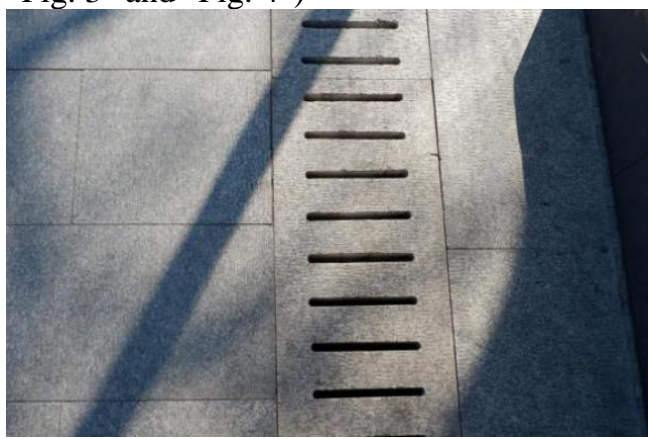

Fig. 3. Pavement of Yangliuqing-style street.

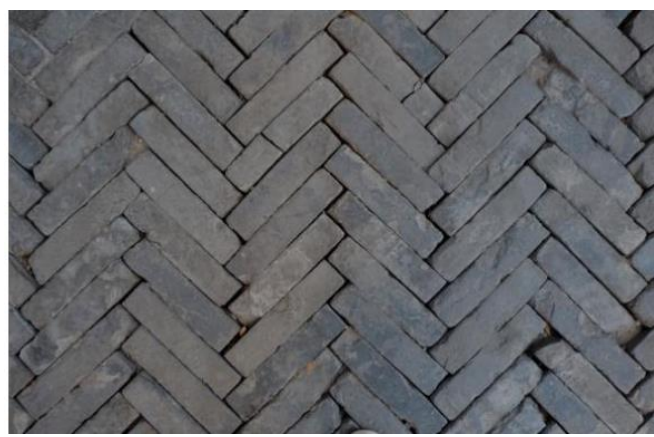

Fig. 4. Pavement of Qiaojiageda alley.

\section{E. Landscape sketch}

1) Landscape wall: The landscape wall is a combination of partitions in landscape design. Not only can it effectively distinguish functions, it also plays a good decorative role in the landscape. Excellent landscape walls can make dull spaces mysterious or ordinary settings lively and interesting. The walls allow the space to be freely hidden, exposed, illuminated, darkened, partitioned, combined and returned, reflecting the essence of the vast Chinese culture. If the landscape design can be carefully and rationally arranged, the effect of the landscape will be more effective and educational.

2) Sculpture: Sculpture is sometimes a landmark in the city. Although it is a decoration to the urban environment, it is highly decorative and easily becomes the focus of vision. The sculptural images in the urban 
landscape can not only reflect the aesthetic orientation and taste of different regions, but also disclose the historical and cultural carrier of the region, and give people beautiful hope. Therefore, the design and construction of the sculpture should meet the aesthetics of most people, and its style should be easy for people to understand. The performances of sculptures are usually metaphorical and symbolic, or sublime or approachable, conveying social themes and positive energy.

3) Seat: In the urban landscape, the seats are not only for people to have a rest, but also the decoration in the garden art, playing the role of the finishing point. Therefore, street design combined seat with Yangliuqing New Year paintings, change the shapes of auspicious patterns. According to the traditional local characteristics of Yangliuqing New Year paintings, the materials used are designed into seats, every part and detail of which are dispersed with painting patterns, so that people can enjoy patterns during the break.

4) Lamps and lanterns: In Tianjin's urban landscape design, lamps are used both for lighting and for decoration. Especially when the Chinese lanterns first appeared, the beautiful lights could present the charming scenery in another manner. The making of lamps is a simple process with low cost. Therefore, the designer is responsible for the effective use of lamps and lanterns. However, it seems that most lamps do not have their own characteristics. If the circular lamp is designed with the traditional pattern elements with regional characteristics, its details, decoration, and other aspects will show rich regional cultural characteristics, which can not only show the traditional emotional appeal of local culture and traditional ethnic patterns, but also play an important role in promoting the image of urban landscape.

\section{F. Plant}

Ancient trees, vines and other plants are important factors in landscape design and are called soft landscapes. In Tianjin, most of the vegetation was later planted artificially. Few of them retain their original features, let alone original ecology. Artificially grown plants falls into only categories: ornamental plants and functional plants. When designing, designers should not only consider their functions, but also fully value the ecological characteristics of the plants themselves, and try to achieve local design, so as to improve the survival rate of the plants. At the same time, seasonal changes must also be considered to ensure all-year scenery, which can also contribute to protection.

\section{G. Water}

Stream water and artificially designed streams play a role in regulating air and expanding space to enhance views. Where there is water, various colorful scenes can be presented, combining the sense of movement and stillness to display the real scene and reflection effect, thus showing the beauty of agility. The water here includes natural water, such as rivers, lakes, and streams, which are products of natural changes. In addition, there are also artificial water, such as fountains, springs, and dry land fountains. The water landscape is both magnificent and exquisite. The design and application of water landscape can better create a dynamic sense of space, and combine it with the surrounding landscape, thus realizing the association of activity and inertia.

\section{CONCLUSION}

The patterns, images, text symbols and deep cultural charm of Yangliuqing New Year paintings can all be the constituent elements of urban landscape design in Tianjin. Designers must find the right language, form, technique, etc., and put them in the right "place", and the cultural tension they exhibit is strong enough. From the perspective of historical development, many vivid and fascinating New Year painting patterns have been handed down to today, which fully proves their tenacity and prosperity.

\section{References}

[1] Yin Yaodong. Classification and Artistic Features of Yangliuqing Woodcut New Year Pictures [J]. Yi Hai. 2014, 11, 67-68. (in Chinese)

[2] Han Bing. A Brief Analysis of Color Features of Yangliuqing New Year Paintings [J]. Art Education. 2010, 09, 132. (in Chinese)

[3] Yang Xiaohui, Zhao Shenghong, Du Lijuan. Application of Mongolian Traditional Patterns in Urban Architecture Landscape Design [J]. Westl Eatbar. 2016, Volume 38, 04, Page 35-36. (in Chinese)

[4] Tang Li. Additional Pattern in Modern Landscape Design [D]. Chongqing University, 2016. (in Chinese)

[5] Zhao Hao, Gao Julan. The Essence of Not Abandoning: a Study on the Innovation and Inheritance of Yi Patterns [J]. Decoration, 2018 (09):130-131. (in Chinese)

[6] Deng Teng. Study on the Extraction and Reconstruction of the Artistic Elements of Flower Pattern in Shaoyang Blue Printing Cloth[D] (in Chinese) 